\title{
Falls during inpatient rehabilitation in spinal cord injury, acquired brain injury, and neurologmusculoskeletal disease programs
}

\author{
Alexander Wilson ${ }^{1,2} \cdot$ Dilnur Kurban ${ }^{3}$ - Vanessa K. Noonan ${ }^{3,4}$ - Andrei Krassioukov 1,5,6 \\ Received: 21 October 2018 / Revised: 14 September 2019 / Accepted: 3 October 2019 / Published online: 22 October 2019 \\ (c) The Author(s), under exclusive licence to International Spinal Cord Society 2019
}

\begin{abstract}
Study design Retrospective chart review.

Objectives To compare the proportion of fallers and the patient level and fall characteristics among inpatients who had experienced at least one fall in a spinal cord injury (SCI), an acquired brain injury (ABI), and a neuromusculoskeletal disease (NMS) rehabilitation program.

Setting Tertiary rehabilitation hospital.

Subjects Inpatients who had experienced at least one fall during rehabilitation.

Methods Patient and fall level variables were extracted from electronic medical records over a 5-year period (January 1, 2011 to January 1, 2016): hospital program, age, sex, Functional Independence Measure (FIM) scores, length of stay, number of medications, as well as fall date, time, location, cause, harm, fall risk assessment data, and whether the fall was witnessed. The impact of hospital program on fall was examined using bivariate and multivariable analysis.

Results Two hundred and thirty-seven (16\%) inpatients experienced at least one fall during the study period. Inpatients with SCI had the highest proportion of fallers (20\%) and fell later after admission than inpatients in the other programs. Patients with $\mathrm{ABI}$ were more likely to sustain moderate-to-severe physical harm from falls. Taking $>5$ medications at time of fall and being earlier in one's rehabilitation course were associated with increased fall rate among fallers.

Conclusions Although the type of program was not a significant predictor of fall rate in the multivariable analysis, there were some important differences among the rehabilitation programs on patient and fall level characteristics. These results may be useful when developing and timing fall prevention interventions for inpatient rehabilitation.
\end{abstract}

Andrei Krassioukov

krassioukov@icord.org

1 International Collaboration on Repair Discoveries, University of British Columbia, Vancouver, BC, Canada

2 MD training program, University of British Columbia, Vancouver, BC, Canada

3 Rick Hansen Institute, Costal Health Authority, Vancouver, BC, Canada

4 Department of Orthopedics, Faculty of Medicine, University of British Columbia, Vancouver, BC, Canada

5 Division of Physical Medicine and Rehabilitation, University of British Columbia, Vancouver, BC, Canada

6 GF Strong Rehabilitation Centre, Costal Health Authority, Vancouver, BC, Canada

\section{Introduction}

Patients undergoing in-hospital rehabilitation following debilitating injury or medical conditions face a significant risk of falling [1]. Inpatient fall rates range from 13 to $14 \%$ in general rehabilitation settings that treat patients with stroke, amputations, SCI, multisystem trauma, and other neuromuscular conditions, and rise to $39 \%$ in geriatric stroke rehabilitation units $[1,2]$. This is far greater than the $1.4 \%$ inpatient fall rate reported in general acute care hospitals $[1,2]$. Falls can lead to injury, decreased quality of life, prolonged hospitalization, and increased costs [1, 3-6]. For this reason, many studies have investigated risk factors for falls in rehabilitation hospitals in order to develop fall prevention programs [1]. Some established risk factors for falling include age, admission Functional Independence Measure (FIM) scores, diagnosis of stroke or amputation, time of day, time in rehabilitation course, history of falls, and polypharmacy [1, 2, 7-9]. For instance, rehabilitation 
inpatients fall more often during daylight hours and it has been shown that those with lower admission FIM scores, increasing age, and those who are earlier on in their rehabilitation course have a higher risk of falling [1,2]. Previous studies have investigated these fall risk factors in general rehabilitation populations but there is a lack of evidence comparing falls between different groups of patients undergoing inpatient rehabilitation (e.g., stroke, SCI, neuromuscular conditions, etc.). The objective of this study was to compare the proportion of fallers as well as the patient level and fall level characteristics among fallers in a spinal cord injury (SCI), an acquired brain injury (ABI), and a neuromusculoskeletal disease (NMS) program, as well as the causes and consequences of the falls. A secondary objective was to examine the sensitivity of the CAMP-V fall risk assessment tool used at our facility that uses medical factors including cognition impairment, anesthesia, altered elimination, mobility impairment, previous falls, and vision impairment to screen for fall risk.

\section{Methods}

This study was conducted in a single tertiary rehabilitation hospital within the Vancouver Coastal Health (VCH) authority in British Columbia, Canada that provides inpatient and outpatient rehabilitation care. We conducted a retrospective study with evaluation of inpatient falls in three inpatients programs (ABI, SCI, and NMS). The SCI program admits both traumatic and nontraumatic SCI; the ABI program admits patients with cerebrovascular accidents and traumatic brain injury; and the NMS program admits patients with multisystem trauma, solid organ transplantations, and neuromuscular disease such as amyotrophic lateral sclerosis, multiple sclerosis, and Guillain-Barré syndrome. The protocol for this study was approved by the Hospital and University Research Ethics Committees.

Analysis of the inpatient falls occurring in the SCI, ABI, and NMS programs over a period of 5 years (January 1, 2011 to January 1, 2016) was conducted using an online VCH hospital incident reporting system. The following information was obtained from this system: hospital program, age, fall date, time, and location, whether a fall was witnessed by hospital staff or not, cause, whether a CAMP$\mathrm{V}$ fall risk assessment was done, and whether the faller was identified as being at risk. The CAMP-V assessment accounts for whether a patient has cognitive impairment, altered bowel or bladder elimination, mobility impairment, previous falls, and impaired vision to predict fall risk. In addition, the patients' degree of harm from the fall was determined by the observing nurse and physicians at the time of the incident and was included in the analysis. Additional patient information was obtained from the patients' electronic medical record including demographic data of each individual, diagnosis, medications taken at the time of fall, and FIM score at admission including motor and cognitive sub scores. The FIM score consists of 13 motor tasks and 5 cognitive tasks and performance is rated on a seven-point scale and it has been shown to be a reliable tool to determine a patient's level of disability [10]. Total FIM score was obtained by summing total FIM motor score and total FIM cognitive scores. The total number of inpatients discharged from the hospital was obtained from admission and discharge records and corrected for service interruptions to ensure patients were only counted once.

Descriptive statistics were used to describe proportion of fallers, fall level information, and patient-level characteristics, for all hospital programs combined and stratified for each program. Bivariate analyses were performed on demographic variables, medications, risk assessment, timing of fall, and FIM scores to determine whether there was a significant difference in these factors among three hospital programs. Continuous variables were compared using ANOVA if they were normally distributed, and using Kruskal-Wallis test if the normality assumption was not met. Categorical data were compared using Chi-squared test unless the expected cell counts were smaller than 5 , in which case Fisher's exact test was used. Receiver operating characteristic (ROC) analysis was carried out to assess the sensitivity of the risk assessment tool. A zero-truncated Poisson regression was run for the full cohort to evaluate the impact of hospital program as well as age at injury, total FIM at admission, number of medications, number of weeks after admission in which a fall occurred and time of fall on the fall rate. Akaike information criterion was used to evaluate and compare models on goodness of fit. Associations with a $p$ value $<0.05$ were considered statistically significant. All analyses were performed using SAS software, Version 9.4 of the SAS System for Windows. Copyright $^{\circledR}$ 2013. SAS Institute Inc., Cary, NC, USA.

\section{Results}

\section{Proportion of fallers}

A total of 1492 patients underwent inpatient rehabilitation within the three programs of the rehabilitation center over the 5-year study period. Of the patient population, 237 inpatients who were fallers were included in this study: $(n=93,39 \%)$ SCI, $(n=97,41 \%) \mathrm{ABI}$, and $(n=47,20 \%$, Table 1) NMS inpatients.

The SCI program had the highest proportion of fallers (20\%), followed by the ABI (18\%) and NMS (10\%) programs. The proportion of fallers was statistically significantly different among three programs, further implying 
Table 1 Patient-level characteristics for the entire cohort $(n=237)$

\begin{tabular}{|c|c|c|c|c|c|}
\hline Variable & Total cohort $n=237$ & SCI $n=9339.2 \%$ & ABI $n=9740.9 \%$ & NMS $n=4719.8 \%$ & $P$ value \\
\hline Age; mean (SD) & $46.2(15.6)$ & $44.5(16.5)$ & $45.2(14.7)$ & $51.5(14.9)$ & 0.03 \\
\hline Male; $n(\%)$ & $152(64.1)$ & $68(73.1)$ & $58(59.8)$ & $26(55.3)$ & 0.06 \\
\hline Total number of falls: median (IQR) & $1.0(1.0)$ & $1.0(1.0)$ & $1.0(1.0)$ & $1.0(1.0)$ & 0.31 \\
\hline Total admission FIM scores: mean (SD) & $71.4(24.8)$ & $71.5(21.4)$ & $62.9(25.7)$ & $90.3(19.0)$ & $<0.0001$ \\
\hline Admission cognitive FIM scores: median (IQR) & $31.0(13.0)$ & $35.0(2.0)$ & $22.0(10.0)$ & $35.0(3.0)$ & $<0.0001$ \\
\hline Admission motor FIM scores: mean (SD) & $43.9(20.4)$ & $39.1(17.7)$ & $42.6(21.1)$ & $57.4(18.9)$ & $<0.0001$ \\
\hline Total discharge FIM scores: median (IQR) & $110(27.5)$ & $114.0(28.0)$ & $102.0(27.0)$ & $112.5(21.0)$ & 0.004 \\
\hline Discharge cognitive FIM scores: median (IQR) & $33.0(7.0)$ & $35.0(0.0)$ & $27.0(9.0)$ & $34.0(3.0)$ & $<0.0001$ \\
\hline Discharge motor FIM scores: median (IQR) & $79.0(22.5)$ & $80.0(25.0)$ & $76.0(22.0)$ & $80.0(20.0)$ & 0.63 \\
\hline Length of stay (days): median (IQR) & $91.0(61.0)$ & $96.0(51.0)$ & $100.0(74.0)$ & $56.0(46.0)$ & $<0.0001$ \\
\hline
\end{tabular}

For normally distributed variables, mean (SD) was reported. For nonnormally distributed variables, median (IQR, interquartile range) was reported

that SCI program had significantly higher proportion of fallers compared with the NMS program.

A total of 351 falls were recorded. Seventy-six patients (32\%) had more than one fall, with three patients having multiple falls on the same day. The number of patients with multiple falls were $(n=35,36 \%)$ in the ABI program, $(n=$ $28,30 \%)$ for the SCI program, and $(n=13,28 \%)$ NMS program, respectively. The median number of falls was 1 [Interquartile range (IQR): 1] and did not differ significantly between programs $(p=0.31$, Table 1$)$, or between patients with nontraumatic SCI and traumatic SCI $(p=0.14)$.

\section{Bivariate analysis}

\section{Patient-level characteristics}

Demographics The average age of the patients included in the cohort was $46 \pm 16$ and differed significantly among programs, highest in the NMS program (52 \pm 15 years), followed by the SCI $(45 \pm 17$ years $)$ and ABI $(45 \pm 15$ years) programs $(p=0.03)$. Although only marginally statistically significant, there were a higher proportion of males in the SCI program $(n=68,73 \%)$ than ABI $(n=58,60 \%)$ and NMS program $(n=26,55 \%)(p=0.06$, Table 1$)$.

FIM scores Total admission FIM scores were significantly different among three inpatient programs, with patients admitted to the NMS program having the highest mean scores $(90 \pm 19)$, that is, the highest functional ability and ABI having the lowest degree of function, with a mean score of $(63 \pm 26, p<0.001)$. Median cognitive FIM scores were lowest in patients with ABI (22, IQR: $10, p<0.0001)$; mean admission motor FIM scores were lowest in SCI patients $(39 \pm 18)(p<0.001$, Table 1$)$.

Total and cognitive FIM scores at discharge were significantly different among three programs, with patients in the SCI program having the highest median total (114,
IQR: $28, p=0.004)$ and cognitive FIM scores (35, IQR: 0, $p<0.0001)$ at discharge. Discharge motor FIM scores did not differ significantly among three programs $(p=0.63$, Table 1$)$.

Length of stay Total length of stays at the rehabilitation center were significantly different among three programs, with patients in the ABI program having the longest median length of stay (100 days, IQR: 74), followed by SCI program (96 days, IQR: 51$)$ and NMS program (56 days, IQR: $46, p<0.0001)$.

\section{Fall level characteristics}

Timing of falls More than half of the falls $(n=192$, $54.7 \%$ ) occurred between 8 a.m. and 4 p.m. There was no difference in frequency of falls in terms of nursing shift times among programs, with most falls $(n=309,88 \%)$ occurring during the first ( 7 a.m. to 3 p.m.) and second ( 3 p.m. to 11 p.m.) nursing shifts $(p=0.76)$. However, the median number of weeks after admission in which a fall occurred differed between programs, being longest in the SCI program ( 7 weeks), followed by the ABI (5 weeks) and NMS ( 3 weeks) programs $(p<0.001$, Table 2$)$.

Similarly, when rehabilitation quintiles (five equal intervals of a patient's entire stay) were looked at, the median quintile of falls in this study was the third quintile. Patients in ABI and NMS programs mostly fell in the second quintile, while patients in the SCI program fell in the third.

Fall location, causes, and degree of harm In all programs combined, most falls occurred on the patient's program location $(n=301,86 \%)$ with the remainder occurring in a therapy location $(n=34,10 \%)$ and dining areas $(n=$ $16,5 \%)$. Patients with SCI had a higher frequency of falls occurring in a therapy location $(n=16,12 \%)$ compared with patients with ABI $(n=15,9 \%)$ and NMS $(n=3,5 \%$, $p=0.04)$. 
Table 2 Fall level characteristics for the entire cohort $(n=351)$

\begin{tabular}{|c|c|c|c|c|c|}
\hline Variable & $\begin{array}{l}\text { Total cohort } \\
n=351\end{array}$ & $\begin{array}{l}\text { SCI } n=132 \\
37.6 \%\end{array}$ & $\begin{array}{l}\text { ABI } n=159 \\
45.3 \%\end{array}$ & $\begin{array}{l}\text { NMS } n=60 \\
17.1 \%\end{array}$ & $P$ value* \\
\hline Time of falls; $n(\%)$ & & & & & 0.92 \\
\hline 00:00-07:59 & $51(14.5)$ & 18 (13.6) & $23(14.5)$ & $10(16.7)$ & \\
\hline 08:00-15:59 & $192(54.7)$ & $72(54.6)$ & $90(56.6)$ & $30(50.0)$ & \\
\hline $16: 00-23: 59$ & $108(30.8)$ & $42(31.8)$ & $46(28.9)$ & $20(33.3)$ & \\
\hline Nursing shifts; $n(\%)$ & & & & & 0.76 \\
\hline $07: 00-14: 59$ & $190(54.1)$ & $72(54.5)$ & $89(56.0)$ & $29(48.3)$ & \\
\hline $15: 00-22: 59$ & 119 (33.9) & $45(34.1)$ & $53(33.3)$ & $21(35.0)$ & \\
\hline 23:00-06:59 & $42(12.0)$ & $15(11.4)$ & $17(10.7)$ & $10(16.7)$ & \\
\hline Number of weeks falls occur after admission: median (IQR) & $5.0(8.0)$ & $7.0(7.0)$ & $5.0(11.0)$ & $3.0(5.0)$ & $<0.0001$ \\
\hline Fall location; $n(\%)$ & & & & & 0.04 \\
\hline Patient floor & $301(85.8)$ & $107(81.1)$ & $142(89.3)$ & $52(86.7)$ & \\
\hline Therapy location (gym pool) & $34(9.7)$ & $16(12.1)$ & $15(9.4)$ & $3(5.0)$ & \\
\hline Cafeteria/dining room & $16(4.5)$ & $9(6.8)$ & $2(1.3)$ & $5(8.3)$ & \\
\hline Witnessed yes; $n(\%)$ & $110(31.3)$ & $39(29.6)$ & $51(32.1)$ & $20(33.3)$ & 0.84 \\
\hline Cause of falls & & & & & 0.08 \\
\hline Using bathroom (toilet, commode, showering, bathing) & $51(14.5)$ & $18(13.6)$ & $22(13.8)$ & $11(18.3)$ & \\
\hline Walking with or without assistance/bending/reaching/leaning & $89(25.4)$ & $27(20.5)$ & $42(26.4)$ & $20(33.3)$ & \\
\hline $\begin{array}{l}\text { Attempting to transfer or sit between bed/stretcher/chair/ } \\
\text { wheelchair }\end{array}$ & $133(37.9)$ & $53(40.2)$ & $67(42.1)$ & $13(21.7)$ & \\
\hline Other & $78(22.2)$ & $34(25.8)$ & $28(17.6)$ & $16(26.7)$ & \\
\hline Degree of harm; $n(\%)$ & & & & & 0.004 \\
\hline No harm & $229(65.2)$ & $76(57.6)$ & $117(73.6)$ & $36(60.0)$ & \\
\hline Minor harm & $112(31.9)$ & $54(40.9)$ & $35(22.0)$ & $23(38.3)$ & \\
\hline Moderate-severe harm & $10(2.9)$ & $2(1.5)$ & $7(4.4)$ & $1(1.7)$ & \\
\hline Number of medications at fall; $n(\%)$ & & & & & 0.74 \\
\hline$\leq 5$ & $279(79.5)$ & $104(78.8)$ & $129(81.1)$ & $46(76.7)$ & \\
\hline$>5$ & $72(20.5)$ & $28(21.2)$ & 30 (18.9) & $14(23.3)$ & \\
\hline
\end{tabular}

* $p$ values were for the comparison of fall characteristics in three programs

The causes of falls did not differ significantly among programs. Transfer related activity was the most common cause $(n=133,38 \%)$ attributed to falls and was followed by walking with or without assistance $(n=89,25 \%)$ and bathroom activities including toileting, showering, and bathing activity $51(n=51,15 \%)$. The remaining $(n=78$, $22 \%$ ) fall causes were listed as "other" within the fall database. About $(n=110,31 \%)$ of falls were witnessed, with similar proportions in three programs $(p=0.84)$.

Most falls resulted in no physical harm among the SCI $(n=76,58 \%)$, ABI $(n=117,74 \%)$, and NMS $(n=36$, $60 \%)$ programs. However, more falls in patients with ABI $(n=7,4 \%)$ resulted in moderate-to-severe harm compared with falls in patients with SCI $(n=2,2 \%)$ and NMS $(n=1$, $2 \%)(p=0.004)$.

Medications The distribution of grouped medications was similar among patients in the three programs, with $80 \%$ of patients $(n=279)$ patients having less than or equal to five medications at the time of the fall $(p=0.74$, Table 2).

Risk assessment At our facility, the CAMP-V risk assessment was performed on $65 \%$ of all inpatients, and $57 \%$ of the assessed patients were identified as being at risk for falls. Subsequent sensitivity analysis of the assessment tool revealed an area under the ROC curve of 0.54 , indicating very poor predictive power of this risk assessment tool.

Multivariable analysis In zero-truncated Poisson regression model on fall rate, the number of medications and number of weeks after admission in which a fall occurred were the only two significant predictors. After adjusting for other variables in the model, patients who have more than five medications fall significantly sooner in their rehabilitation stay compared with patients with five or less medications at the time of fall $(p=0.02)$. Similarly, patients fall significantly soon after admission compared 
Table 3 Zero-truncated Poisson regression of fall rate among fallers

\begin{tabular}{|c|c|c|c|c|c|}
\hline \multirow[t]{2}{*}{ Independent variables } & \multirow[t]{2}{*}{ Coefficients } & \multirow[t]{2}{*}{ Standard error } & \multicolumn{2}{|c|}{$\begin{array}{l}95 \% \\
\text { confidence limits }\end{array}$} & \multirow[t]{2}{*}{$P$ value } \\
\hline & & & Lower & Upper & \\
\hline Intercept & -3.55 & 0.47 & -4.46 & -2.63 & $<0.0001$ \\
\hline Age & 0.0003 & 0.006 & -0.01 & 0.01 & 0.96 \\
\hline Total FIM at admission & -0.01 & 0.004 & -0.01 & 0.002 & 0.12 \\
\hline \multicolumn{6}{|l|}{ Number of medications } \\
\hline$\leq 5$ & -0.45 & 0.19 & -0.83 & -0.08 & 0.02 \\
\hline$>5$ & Baseline & & - & - & - \\
\hline Weeks & -0.1 & 0.02 & -0.14 & -0.06 & $<0.0001$ \\
\hline \multicolumn{6}{|l|}{ Identifying program } \\
\hline ABI inpatient & -0.04 & 0.21 & -0.45 & 0.36 & 0.83 \\
\hline NMS inpatient & -0.32 & 0.31 & -0.93 & 0.30 & 0.32 \\
\hline SCI inpatient & Baseline & - & - & - & - \\
\hline \multicolumn{6}{|l|}{ Time of fall } \\
\hline 00:00-07:59 & 0.44 & 0.30 & -0.14 & 1.02 & 0.14 \\
\hline $08: 00-15: 59$ & 0.21 & 0.20 & -0.19 & 0.61 & 0.30 \\
\hline $16: 00-23: 59$ & Baseline & - & - & - & - \\
\hline
\end{tabular}

The $p$ value for the whole model was $<0.0001$, meaning that the model was significantly better than an intercept only model. $N=232$ used for the model; coefficients are log counts of number of falls with near discharge $(p<0.0001$, Table 3$)$. The program (SCI, ABI, and NMS) which is the predictor of interest in this study was not a significant predictor of the fall rate among fallers.

\section{Discussion}

In this study, it was observed that the SCI program had a higher proportion of fallers (20\%) compared with the ABI program (18\%) and NMS program (10\%). This contrasts with previous reports that show that patients with traumatic SCI had lower fall incidences compared with patients with ABI who had a history of stroke $[1,2]$. The SCI program included primarily patients with traumatic SCI (75\%) and there was no difference in the fall rate between those with traumatic and nontraumatic injuries. As previous research is based out of the United States and this study takes place in a Canadian facility, differences in models of care and admission times may account for this discrepancy in proportion of fallers.

The distribution of degree of harm was significantly different among three programs, with higher rates of minor harm observed in the SCI program and moderate-to-severe harm the ABI program. The reason for this difference is not clear. No previous studies have compared injuries between these patient populations. We hypothesize that the ABI program has a higher injury rate because there was a higher proportion of patients who are ambulatory in the ABI program and have greater likelihood to fall from standing height than patients with SCI and NMS who are more likely to fall from a wheelchair or stretcher. Data exploration showed that in our cohort, ambulatory patients were more likely to have minor or moderate-severe injuries than wheelchair users, which makes our hypothesis plausible.

The FIM measure has been shown to be reliable measure to determine a patient's level of disability [10]. Multivariable analyses on the fall rate among fallers did not identify that total FIM score on admission was significantly associated with fall rate. This result was different from other studies which reported that a higher FIM scores were associated with lower fall risk [1, 11]. In our study the fall rate was modeled among fallers, which could be different from modeling fall risk with nonfallers included. The total FIM scores at admission differed between programs with patients admitted to the ABI and SCI having significantly lower FIM scores than NMS patients. This may contribute to the higher fall rates seen in the two programs compared with the NMS program.

Most literature defines polypharmacy as the concurrent use of greater than five medications by a patient $[8,12,13]$. The risk of falling increases with increased medication use due to increased risk of interaction and adverse events $[12,14]$. Furthermore, central nervous system acting drugs, cardiovascular drugs, anticholinergics, and opioid analgesics have been associated with increased fall risk $[8,12,14$ 17]. Given that most patients commonly take these medications, we included the total number of medications in each category that patients were taking in our multivariable analysis. The analysis showed that taking fewer than five 
medications belonging to those categories was associated with reduced the fall rate. Although not statistically significant, on average, patients with SCI were taking slightly more medications at the time of the fall compared with the other two programs and this may partly account for the high fall rate seen in the SCI program. It is unclear as to whether this observed increase in fall risk is due to increased illness severity in patients requiring more medications or due to the medication effects.

Lastly, the multivariable analysis showed that the number of weeks after admission was inversely related to the fall rate among fallers; that is, patients are at higher risk of falling sooner after admission to rehabilitation. This is consistent with previous reports finding that most falls occur within a few weeks of admission [1, 2]. We found that falls occurred a median of 5 weeks after admission, with significant differences existing between programs. Patients with SCI were more likely to fall later in their rehabilitation course with a median fall time of 7 weeks after admission whereas patients on the ABI and NMS had a median fall time of 5 and 3 weeks, respectively. This difference may be because there is a longer delay between admission and beginning active rehabilitation in the SCI program compared with the other programs.

Finally, as part of our study we investigated the use and effectiveness of the CAMP-V falls risk admission assessment used at our facility. We found that $67 \%$ of patients were assessed at admission but only $62 \%$ of those assessed were correctly identified as being at risk for falls. Subsequent sensitivity analysis of the tool revealed an area under the ROC curve of 0.54 , indicating a poor predictive power. This has prompted a review of risk assessment practices in our facility.

There are several limitations in this study that should be noted. First, a large portion of data was extracted from incident reports that were filled out by clinical staff as part of care so the data quality may not be as high as if it was done as part of a prospective research study. Secondly, because the study investigated only patients who fell and does not include nonfallers, cause-effect relationships cannot be identified with certainty and this should be addressed in future research.

In conclusion, we found that the proportion of fallers, fall characteristics, and the patient-level factors that affect fall rate among fallers during inpatient rehabilitation are similar to previous studies. Multivariable analysis revealed that taking more than five medications at the time of fall and being earlier in one's rehabilitation course were associated with an increased fall rate. Although the type of program was not a significant predictor of fall rate among fallers in the multivariable analysis, there were some important differences among the rehabilitation programs on patient and fall level characteristics. Patients with SCI had a higher proportion of fallers and fall later in their rehabilitation course. Patients with ABI were more likely to sustain moderate-to-severe physical harm from falls. The results indicate that the admitting diagnosis may be useful when developing and timing fall prevention interventions and this is an important consideration for rehabilitation administrators and clinicians.

\section{Data availability}

The datasets generated and analyzed during the current study are available from the corresponding author on reasonable request.

Acknowledgements AW is a medical student at the University of British Columbia (UBC). He participated in this project as a part of his Flexible and Enhanced Learning (FLEX) UBC course. This project was completed under the supervision and with the support of AK. The authors are thankful to Ms Carol Chao and Ms Lisa Kawazaki for providing support and encouragement during this study and for Carly Rivers for her review of this paper.

Funding This research project was supported by funding from the University of British Columbia Faculty of Medicine Summer Student Research Program (AW). The Physician-led Quality Improvement Initiative, Specialist Services Committee: a partnership of doctors of BC and the Ministry of Health Canada (AK).

Author's contributions AW was responsible for writing the protocol and proposal, conducting a literature review, extracting and analyzing data, interpreting results, and writing the paper. DK was responsible for statistical analysis of the data and writing the methods and results section of the paper. VN provided input on the analysis plan and edited the paper. AK supervised the project, connected the team with resources, guided the study design and analysis, and provided feedback on the paper.

\section{Compliance with ethical standards}

Conflict of interest The authors declare that they have no conflict of interest.

Ethical approval This study was approached by the University of British Columbia Clinical Research Ethics Board (CREB) (reference number H16-00792-A001). We certify that all applicable institutional and governmental regulations concerning the ethical use of human data were followed during the course of this research.

Publisher's note Springer Nature remains neutral with regard to jurisdictional claims in published maps and institutional affiliations.

\section{References}

1. Lee JE, Stokic DS. Risk factors for falls during inpatient rehabilitation. Am J Phys Med Rehabil. 2008;87:341-50.

2. Vlahov D, Myers AH, al-Ibrahim MS. Epidemiology of falls among patients in a rehabilitation hospital. Arch Phys Med Rehabil. 1990;71:8-12. 
3. Ross MK, Egan E, Zaman M, Aziz B, Dewald T, Mohammed S. Falls in the inpatient rehabilitation facility. Phys Med Rehabil Clin North Am. 2012;23:305-14.

4. Forrest G, Chen E. Efficient assessment of risk of fall. Rehabil Nurs J. 2016;41:320-5.

5. Rochat S, Monod S, Seematter-Bagnoud L, Lenoble-Hoskovec C, Bula CJ. Fallers in postacute rehabilitation have worse functional recovery and increased health services use. J Am Med Dir Assoc. 2013;14:832-6.

6. Wong JS, Brooks D, Mansfield A. Do falls experienced during inpatient stroke rehabilitation affect length of stay, functional status, and discharge destination? Arch Phys Med Rehabil. 2016;97:561-6.

7. Mion LC, Gregor S, Buettner M, Chwirchak D, Lee O, Paras W. Falls in the rehabilitation setting: incidence and characteristics. Rehabil Nurs. 1989;14:17-22.

8. Chiu M, Lee H, Hwang H, Wang S, Lin M. Medication use and fall-risk assessment for falls in an acute care hospital. Geriatrics Gerontol Int. 2015;15:856-63.

9. Frisina PG, Guellnitz R, Alverzo J. A time series analysis of falls and injury in the inpatient rehabilitation setting. Rehabil Nurs J. 2010;35:141-6.

10. Stineman MG, Shea JA, Jette A, Tassoni CJ, Ottenbacher KJ, Fiedler R, et al. The Functional Independence Measure: tests of scaling assumptions, structure, and reliability across 20 diverse impairment categories. Arch Phys Med Rehabil. 1996; 77:1101-8

11. Saverino A, Benevolo E, Ottonello M, Zsirai E, Sessarego P. Falls in a rehabilitation setting: functional independence and fall risk. Eur Medicophysica. 2006;42:179-84.

12. Lamis RL, Kramer JS, Hale LS, Zackula RE, Berg GM. Fall risk associated with inpatient medications. Am J Health Syst Pharm. 2012;69:1888-94.

13. Gacto-Sanchez M, Medina-Mirapeix F, Navarro-Pujalte E, Escolar-Reina P. Changes in disability levels among older adults experiencing adverse events in postacute rehabilitation care: a prospective observational study. Medicine. 2015;94:e570.

14. Zia A, Kamaruzzaman SB, Tan MP. Polypharmacy and falls in older people: balancing evidence-based medicine against falls risk. Postgrad Med. 2015;127:330-7.

15. Zia A, Kamaruzzaman S, Myint PK, Tan MP. Anticholinergic burden is associated with recurrent and injurious falls in older individuals. Maturitas. 2016;84:32-37.

16. Leipzig RM, Cumming RG, Tinetti ME. Drugs and falls in older people: a systematic review and meta-analysis: I. Psychotropic drugs. J Am Geriatr Soc. 1999;47:30-39.

17. Dauphinot V, Faure R, Omrani S, Goutelle S, Bourguignon L, Krolak-Salmon P, et al. Exposure to anticholinergic and sedative drugs, risk of falls, and mortality: an elderly inpatient, multicenter cohort. J Clin Psychopharmacol. 2014;34:565-70. 